\title{
APRECIACIONES SOBRE EL DISEÑO COLABORATIVO DE REDES DE DATOS MEDIANTE TIC
}

\section{INSIGHTS ON COLLABORATIVE NETWORK DESIGN DATA USING ICT}

\author{
José Luis Soto-Ortiz; jlso.uv@gmail.com \\ Carlos Arturo Torres-Gastelú; ctorres@uv.mx \\ Universidad Veracruzana
}

\section{RESUMEN}

Dentro de los programas de modernización de la Universidad Veracruzana en cuanto a su modelo educativo integral y flexible (MEIF), contempla el proyecto AULA cuyo objetivo primordial es el de potenciar una mejora continua de innovación institucional centrada en la práctica docente. En este sentido, el presente trabajo exploratorio, analiza la experiencia de la aplicación de las TIC en la docencia universitaria en la asignatura de redes de datos II. El objetivo es conocer la percepción de los estudiantes, mediante la inclusión de actividades bajo el enfoque del trabajo colaborativo. Para ello, se utilizó el software de diseño de redes y posterior a ello se aplicó la configuración realizada en hardware especializado de red. La recolección de datos, se realizó por medio de una encuesta de tipo mixto, y se aplicó a un grupo de 16 estudiantes que llevó la experiencia. Finalmente los resultados señalan que en el grupo existe una tendencia favorable hacia el uso de las TIC como apoyo complementario en sus asignaturas.

PALABRAS CLAVE: Colaboración, enseñanza virtual, trabajo colaborativo, educación superior.

\section{ABSTRACT}

Within the modernization programs of the Universidad Veracruzana in their comprehensive and flexible educational model (MEIF), included in the project AULA whose primary objective is to promote the continuous improvement of institutional innovation centered teaching. In this sense, the present exploratory study examines the experience of the application of ICT in university teaching in the subject of data networks II. The goal is to understand the perceptions 
of students, by including activities under the collaborative approach work. For this, the network design software and post the settings made in this specialized network hardware used was applied. Data collection was conducted through a survey of mixed type, and applied to a group of 16 students who took the experience. Finally, the results indicate that there is a particular way in the group a favorable towards the use of ICT as additional support in their subjects trend.

KEYWORDS: Collaboration, virtual learning, collaborative work. Higher education.

\section{INTRODUCCIÓN}

La Universidad Veracruzana (UV), se encuentra en un proceso de actualización y modernización de su modelo educativo integral y flexible (MEIF), entre los que destaca el proyecto AULA, cuyo objetivo es el de potenciar una mejora continua de innovación institucional centrada en la práctica docente, teniendo como base el diseño curricular basado en el enfoque de competencias; promoviendo a su vez la formación de redes de comunidades y académicos, centradas en el desarrollo del aprendizaje de los estudiantes y en la construcción colectiva del conocimiento. El documento AULA (2009) tiene como eje rector, la inclusión de aquellas tecnologías que posibiliten el trabajo colaborativo y la construcción social del conocimiento; así como mantener una actualización en el manejo de aquellos recursos tecnológicos que se puedan incorporar en la práctica docente para incorporarlos en el proceso de enseñanzaaprendizaje.

Dentro de este contexto, está situada la Facultad de Estadística e Informática (FEI) perteneciente a la UV, apostando en la formación de profesionales altamente capacitados en el uso y aplicación de las TIC. De acuerdo con el plan de estudios de la licenciatura de informática, se encuentran las Experiencias Educativas (EE) de redes I, II y III relacionadas con el diseño, configuración y administración de redes de datos de área local y amplia. Dichas asignaturas, se han alineado pedagógicamente con el programa académico de Cisco (Netacad) ${ }^{1}$ orientado a la certificación $\mathrm{CCNA}^{2}$ la cual es reconocida en todo el mundo. En este sentido en la FEI, se han utilizado distintas metodologías docentes para la enseñanza, entre los que destacan: a) aprendizaje basado en proyectos, b) técnicas de procesamiento de la información y c) el trabajo colaborativo.

De modo que, el objetivo final tanto para las instituciones educativas como para los docentes, es encaminar los esfuerzos en el desarrollo de competencias en el uso de las TIC, fomentando una cultura digital mediante el reforzamiento de prácticas y hábitos de interacción; pero sobre todo mantener una actitud de colaboración, que coadyuven a los sujetos a mejorar su calidad de vida en el contexto de la sociedad del conocimiento (Orta y Ojeda, 2009). Al respecto, Romero y Guitert (2012) sostienen que las competencias adquiridas en un entorno de

\footnotetext{
${ }^{1}$ El programa académico Netacad es propiedad de la empresa Cisco Inc.

${ }^{2}$ Cisco Certified Networking Assosciate (CCNA) es la certificación de asociado de redes avalado por Cisco.
} 
aprendizaje colaborativo mediado con tecnología, propicia la construcción del conocimiento entre un conjunto de sujetos, como resultado de la interacción entre los mismos para alcanzar un objetivo común. En este sentido, algunos investigadores como Echazarreta, Prados, Poch y Soler (2009); Villalustre y Del Moral (2011) han valorado las habilidades que se desarrollan al incluir en el proceso de aprendizaje el trabajo colaborativo con las TIC.

\section{TRABAJO COLABORATIVO MEDIADO POR TIC}

Con base en lo expuesto, se parte de los referentes acerca del trabajo colaborativo mediado por

TIC. Peñaloza y García (2008) explican que la colaboración implica el uso de una metodología, a través de la cual los estudiantes trabajan conjuntamente, con el fin de resolver actividades de aprendizaje. Se distingue del enfoque cooperativo en que el rol del docente es más protagónico, conduce al grupo con mayor liderazgo, y se asegura de que los estudiantes realicen su parte correspondiente para lograr un objetivo compartido. En cambio, en el enfoque colaborativo, el docente solamente funge como un facilitador y el grupo asume la responsabilidad de trabajar colectivamente en el cumplimiento de sus metas y objetivos.

De manera que el trabajo colaborativo ha sido expuesto desde diferentes posturas que consideran el impacto de la cognición social, el cual reside en la co-construcción y negociación de conocimiento (Peñalosa, García y Espinosa 2008). De acuerdo, con los aportes de Villalustre y Del Moral (2010) señalan que el proceso de aprendizaje está íntimamente ligado a la experiencia de ser parte de una comunidad. Es por ello, que no se debe considerar al estudiante como un ente aislado, al contrario debe estar situado en interacción con los demás miembros de la comunidad de aprendizaje de la que forma parte. De modo que el docente, debe propiciar la interacción en pro de la solución de problemas de manera conjunta, para ello debe crear espacios sociales colaborativos. Asimismo, debe alejarse del papel de transmisor de la información y que sólo promueve el aprendizaje individualizado. De manera que el aprendizaje colaborativo, es la expresión más representativa del socio constructivismo, ya que resalta las líneas teóricas de la interacción sociocognitiva y de la coordinación entre estudiantes.

En un estudio por Roselli (2011), incluyó la corriente tradicional del aprendizaje cooperativo, pero sumó aportes neo-piagetianos como la teoría del conflicto socio-cognitivo; así mismo insertó fundamentos de la teoría de la cognición distribuida desembocando en el enfoque del aprendizaje colaborativo mediado por computadora. En otras palabras, las fuentes básicas del enfoque del trabajo colaborativo son: a) el socio-constructivismo o teoría del conflicto socio cognitivo, b) el enfoque de la intersubjetividad y c) el modelo de cognición distribuida. Estas tres corrientes, se sitúan en un eje individual-grupal, según el énfasis que se ponga en: el individuo, en la interacción o en lo colectivo como tal.

Por su parte, Guerra (2008) explica que el enfoque del trabajo colaborativo mediado por TIC, es una estrategia didáctica, enfatizando la formación del sujeto que aprende como persona en interacción con otras. Esta posición coincide con la afirmación de que la esencia educativa, es la 
esencia del desarrollo de la actividad mental de los seres humanos, como resultado de la socialización, entendiéndose como un proceso de desarrollo de la persona que se da en grupo. Sí la comunicación con el grupo, se desarrolla en la mente de la persona, fomenta la habilidades de trabajo de grupo y responde a la forma de trabajo que se prevé puede ser utilizado en los próximos años, entonces los procesos educativos darán un giro, y pasar del enfoque del aprendizaje individual al aprendizaje en grupo.

Romero y Guitert (2012) afirman que el uso de las TIC radica en lo social, donde los ambientes virtuales de aprendizaje son el contexto ideal para el trabajo colaborativo; por tanto es imperante resolver didácticas acordes con las tecnologías y diseñar mecanismos para su evaluación. Un aspecto importante, radica en la formación de los grupos o equipos de trabajo Johnson y Johnson (1989) señalan que en el empleo de la didáctica del enfoque colaborativo, se recomienda la conformación de grupos reducidos entre 5 o 6 integrantes. En tanto, Webber y Webber (2012) concluyen que los agrupamientos a través de mecanismos automáticos no inciden negativamente en la efectividad de la colaboración en los participantes de la misma, ya que esto resulta un factor decisivo y garantiza el éxito del trabajo del grupo.

Soto y Torres (2013) afirman que la colaboración en el ámbito universitario, propicia algunas de las ventajas entre las que destacan: el compartir información, crear el conocimiento colectivo y mejorar el proceso de enseñanza-aprendizaje en equipo. Asimismo, dicho enfoque, favorece las aportaciones de la comunidad de aprendizaje de la cual forma parte, para que el individuo adquiera nuevas habilidades. Esto, requiere que se promuevan las competencias necesarias en vías de la creación de comunidades de aprendizaje. Aprovechando, las herramientas tecnológicas disponibles, tales como wikis, blogs, foros, etc. Estas herramientas tecnológicas hacen posible la interacción entre los individuos, de tal suerte que el aprendizaje puede considerarse un proceso extrínseco al estudiante, mismo que aprovecha las sinergias de la comunidad de aprendizaje para construir el conocimiento (Villalustre y Del Moral, 2010).

\section{RECURSOS TIC}

Si bien la tecnología, sirve de apoyo al docente en el diseño y planificación para el desarrollo de su clase, con una estrategia didáctica adecuada también puede ser una herramienta que le facilite la interacción con sus estudiantes. En este plano, las TIC benefician el logro del trabajo colaborativo, principalmente aquellas aplicaciones que pueden ser utilizadas en las tareas académicas y que algunos autores han denominado como herramientas web 2.0 (Basilotta y Herrada, 2013). Calzadilla (2008) sugiere que las TIC representan ventajas para el trabajo colaborativo, porque estimulan la comunicación interpersonal al facilitar el intercambio de información y el diálogo entre los sujetos implicados en el proceso. En relación con el estudiante, las TIC contribuyen a facilitar el trabajo colaborativo en un doble sentido: por un lado, fomentando su desarrollo individual, y por otro, estimulando la interacción educativa con sus compañeros del grupo de trabajo. 
Para el desarrollo y seguimiento de la EE de Redes II, se cuentan con diversas tecnologías de la información y comunicaciones empleadas para este fin. Cabe señalar, que el proceso de selección de las herramientas tecnológicas, se realizó con antelación al inicio del curso y responde al objetivo general de la EE. Para el área de Redes, es de gran utilidad el software que ofrece la empresa Cisco, en apoyo a la academia Netacad, entre los aplicativos de desarrollo que distribuye, se encuentra el simulador de redes denominado Packet $\operatorname{Tracer}^{3}$ (PT), ver Figura 1.

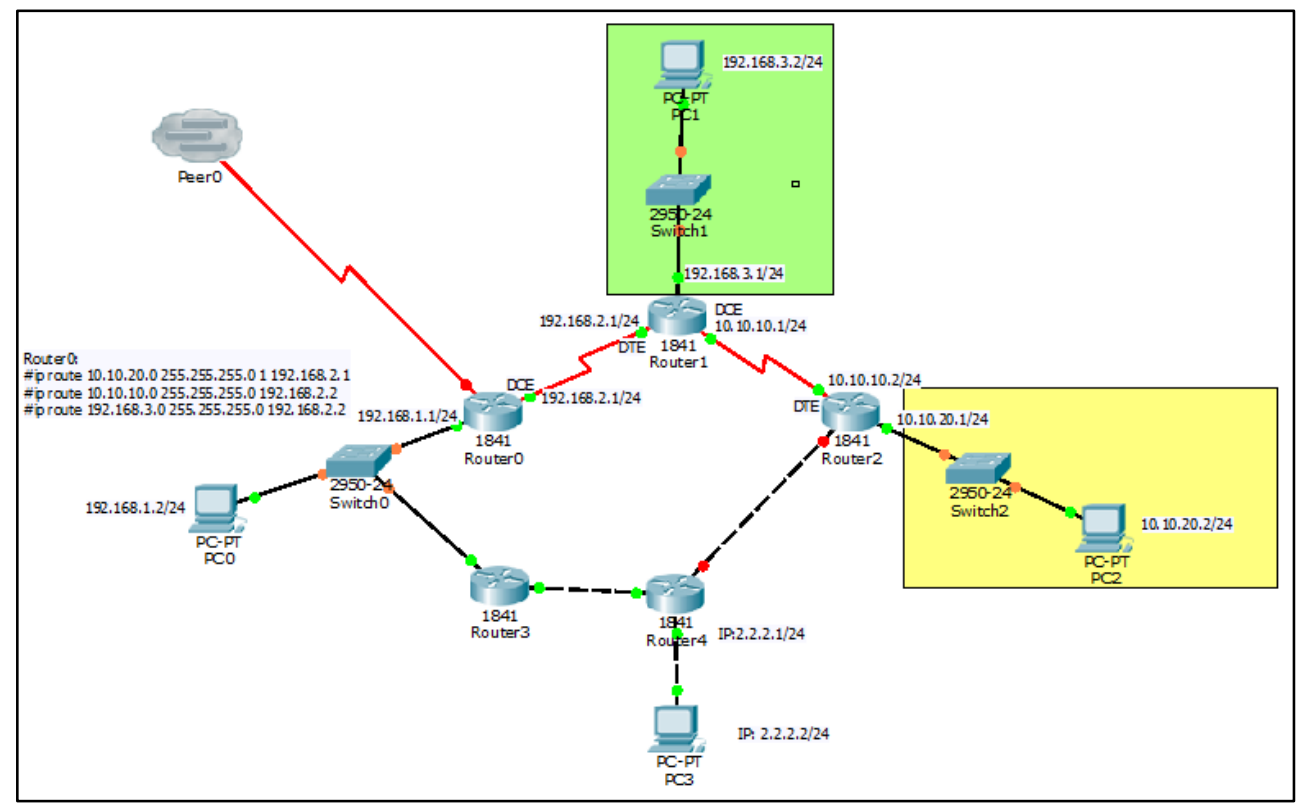

Figura 1. Ejemplo de topología de red en PT. Elaboración propia.

Entre las características que destacan el PT, es un programa informático que se instala de manera local en una computadora, este aplicativo se utiliza para el diseño, emulación y configuración de topologías de equipos de red. Permite al estudiante, aplicar la simulación de escenarios de red mediante prácticas previamente diseñadas, sin la necesidad de contar con equipo físico especializado de red. Asimismo, desarrolla las actividades de aprendizaje relacionadas con la configuración de los dispositivos de red, por medio de la animación y el seguimiento de paquetes. Con estas prácticas, el estudiante aplica los conocimientos y habilidades adquiridos previamente en clase, para la implementación de los distintos protocolos utilizados en las redes de datos como: IPv4, RIPv1 y 2, EIGRP, OSPF, TCP, UDP, entre otros. Asimismo, el PT cuenta con un módulo de multiusuario (multiuser en inglés), el cual permite la conexión entre instancias de PT instaladas en diferentes computadoras e interconectadas en una red local. Lo anterior, amplifica las posibles combinaciones de escenarios, llevando las prácticas de un aprendizaje individual situado, a un trabajo colaborativo en red.

\footnotetext{
${ }^{3}$ El software Packet Tracer es un programa para computadoras desarrollado por Cisco.
} 
Para la comunicación y reflexión de los temas vistos en clase y en las prácticas, se utilizó el aula virtual denominada Eminus $^{4}$ (véase la Figura 2). La plataforma para gestión del aprendizaje (EMINUS) fue desarrollado por la Universidad Veracruzana (UV), integra diferentes aplicaciones entre las que destacan: a) foros, b) wikis, c) manejo de contenidos, d) intercambio de archivos, e) blogs y e) seguimiento de los estudiantes. Esta plataforma, posibilita el aprendizaje en línea, para ello se crearon los foros de debate, relacionadas con las temáticas de las actividades didácticas realizadas en clase. Finalmente, se cuenta con hardware especializado de red (switches y routers) en donde los estudiantes desarrollan sus prácticas de manera colaborativa y aplican sus conocimientos adquiridos, teniendo escenarios reales.

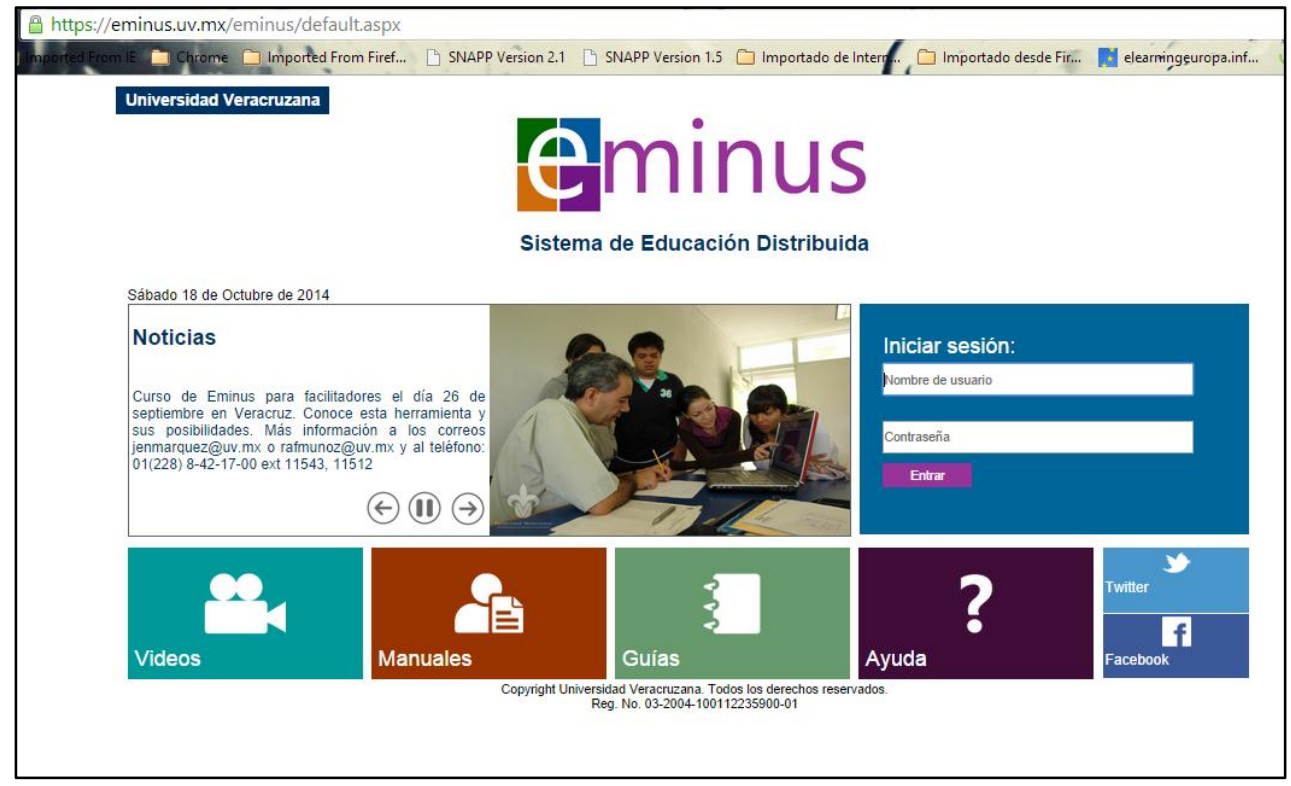

Figura 2. Aula virtual Eminus. Elaboración propia.

\section{METODOLOGÍA}

\section{Propósito del estudio}

El objetivo de este estudio, fue conocer la apreciación de un grupo de estudiantes acerca de la inclusión de actividades de aprendizaje bajo el enfoque del trabajo colaborativo, mediante el uso de las TIC.

\section{Participantes}

La muestra de este estudio, se define como no probabilística e intencional, por ser la que más se ajusta al objetivo del estudio de la aplicación del trabajo colaborativo en grupos reducidos en

\footnotetext{
${ }^{4}$ El aula virtual Eminus, es una plataforma de administración de contenidos de aprendizajes desarrollado por la U.V.
} 
cuanto a integrantes y por tratarse de un estudio exploratorio. Los criterios de inclusión de los participantes fueron: (1) Facultad de Estadística e Informática; (2) Licenciatura en informática; (3) Estudiantes que cursaron la asignatura de Redes II; (4) Semestre lectivo de 2013-2014; (5) No aplica distinción de género. A partir de ello, la muestra quedó conformada por 16 estudiantes de los cuales $70.4 \%$ corresponden al sexo masculino y $29.6 \%$ al sexo femenino, en un rango de edades entre los 19 a 24 años.

\section{Actividad didáctica}

El objetivo final de esta experiencia educativa, fue aplicar los conocimientos adquiridos en clase, en la que los estudiantes recibieron los fundamentos teóricos para el diseño de topologías y la configuración de los equipos de red. Con respecto a las clases presenciales, alternaron la tutoría docente con las actividades de los discentes como: debates, análisis de los módulos y las prácticas a realizar sobre el contenido de la asignatura.

Por su parte, el alumno aplicó los aspectos teóricos mediante la realización de prácticas basadas en el enfoque del trabajo colaborativo, para ello se apoyó en el uso de tecnologías digitales, en este caso el programa de simulación de redes Packet Tracer aplicando el módulo de "multiusuario" con la finalidad de interconectar mediante red otras instancias de Packet Tracer y así trabajar de forma colaborativa.

La intervención pedagógica, se focalizó en las 11 unidades temáticas de la asignatura combinando la teoría y la práctica, tanto en las actividades presenciales como en las virtuales. Para la realización de los debates grupales, se activaron foros de discusión en el aula virtual en Eminus. De igual forma, en cada módulo se incluyeron los objetivos, un esquema de trabajo, la introducción, teoría y ejemplos de cada tema con presentaciones en Power Point y en video. Finalmente, se consideraron las prácticas que incluían los ejercicios a realizar tanto en el PT como en los equipos de red.

Con respecto a la evaluación del curso, se le ponderó de la siguiente manera: $40 \%$ correspondió al examen teórico escrito y un $60 \%$ a las actividades en línea y prácticas realizadas. La descripción de los módulos, la modalidad y recurso empleado (véase Tabla 1).

Para realizar lo anterior, los estudiantes trabajaron diferentes etapas:

- La primera, se basó en actividades de trabajo individual, pero con participación activa en el intercambio de mensajes, para ello se utilizó un foro en la plataforma Eminus, en el cual cada estudiante debatió acerca de los temas relacionados con la configuración de equipos de red, así como los sub-temas de enrutamiento estático y dinámico.

- La segunda etapa, consistió en la inserción del trabajo colaborativo en donde los estudiantes realizaron diversas topologías de red, utilizando el PT y mediante actividades de multiusuario, que permitió conectar las instancias de PT en la red local.

- Como tercer momento y etapa final, se implantó la configuración, conexión y puesta en marcha de la topología realizada en PT, pero aplicado en los equipos de red (routers y 
switches). En dicho proceso, los estudiantes se repartieron las actividades considerando la cantidad de equipos con los que se contaban y cada uno realizó la configuración de los equipos de red que les tocaron. Todo este proceso estuvo acompañado bajo la supervisión tutorial docente.

\begin{tabular}{|c|l|l|l|}
\hline Módulo & \multicolumn{1}{|c|}{ Nombre } & Modalidad & \multicolumn{1}{|c|}{ Recurso empleado } \\
\hline 1 & $\begin{array}{l}\text { Introducción al enrutamiento y envío } \\
\text { de paquetes }\end{array}$ & Individual & Eminus \\
\hline 2 & Enrutamiento estático & Individual & Eminus \\
\hline 3 & $\begin{array}{l}\text { Introducción a los protocolos de } \\
\text { enrutamiento dinámico }\end{array}$ & Individual & Eminus \\
\hline 4 & $\begin{array}{l}\text { Protocolos de enrutamiento por } \\
\text { vector-distancia }\end{array}$ & Individual & Eminus \\
\hline 5 & RIPv1 & $\begin{array}{l}\text { Colaborativ } \\
\text { o }\end{array}$ & $\begin{array}{l}\text { Eminus, PT y Equipos de } \\
\text { red }\end{array}$ \\
\hline 6 & VLSM y CIDR & $\begin{array}{l}\text { Colaborativ } \\
\text { o }\end{array}$ & $\begin{array}{l}\text { Eminus, PT y Equipos de } \\
\text { red }\end{array}$ \\
\hline 8 & RIPV2 & $\begin{array}{l}\text { Colaborativ } \\
\text { o }\end{array}$ & $\begin{array}{l}\text { Eminus, PT y Equipos de } \\
\text { red }\end{array}$ \\
\hline $9-11$ & EIGRP & $\begin{array}{l}\text { Colaborativ } \\
\text { o }\end{array}$ & $\begin{array}{l}\text { Eminus, PT y Equipos de } \\
\text { red }\end{array}$ \\
\hline
\end{tabular}

Tabla 1. Programa académico de la Experiencia Educativa Redes II. Elaboración propia.

\section{Instrumento}

Para la recolección de datos, se diseñó un cuestionario de tipo mixto denominado colab-uv diseñado exprofeso, el cual se conformó por 4 ítems de identificación personal y 16 ítems agrupados en dos indicadores (percepción y actitud), que permiten valorar la colaboración mediada por TIC por parte de los estudiantes. En la mayoría de los cuestionamientos, se definieron parámetros de valor basados en la escala de Likert de cuatro valores: determinante, significativo, poco y nulo. El cuestionario, se diseñó mediante la aplicación de Forms del googledocs. Además, se hizo llegar por medio de correo electrónico adjuntando el url del instrumento, esto permitió que el cuestionario fuese respondido desde cualquier lugar teniendo conexión a Internet, una vez con los datos almacenados, se realizó el tratamiento de la información mediante el programa SPSS.

La dimensión de la construcción colaborativa del conocimiento, se conformó por el cuestionamiento: ¿En qué grado, son importantes para ti los siguientes aspectos en la construcción colaborativa del conocimiento?

- El sentido de la colaboración. 
- Principal motivación al iniciar un trabajo colaborativo.

- Empatía.

- El compromiso individual.

- El compromiso grupal.

- La tolerancia.

- Confianza en los demás.

- El respeto a la diversidad.

- Manejo asertivo del error.

Con respecto a la valoración de la percepción, tiene que ver con la actitud de los estudiantes una vez que realizaron las actividades colaborativas, los cuestionamientos son los siguientes:

- Considero que las herramientas web de tipo social son importantes para mi aprendizaje en el momento actual

- Me siento a gusto realizando tareas grupales.

- La responsabilidad individual y en equipo es positiva para el logro de la tarea.

- La crítica en el grupo me ayudó a mejorar mis aportaciones.

- Las herramientas sociales no me favorecen en el trabajo en grupo.

- Me agobia la participación en foros.

- Me parece conveniente introducir la colaboración en la enseñanza.

- Me motiva el trabajo grupal, siempre aprendo de las experiencias de otros.

\section{Análisis de confiabilidad}

Para valorar la confiabilidad del cuestionario se aplicó una prueba piloto a un grupo de 80 estudiantes, obteniendo un coeficiente de consistencia interna alfa de 0,870 de Cronbach, este valor al estar en un rango cercanos al 0.90 , se considera el índice de fiabilidad como aceptable. De acuerdo con Bisquerra (2004) los valores de alfa situados entre el intervalo de 0.8 a 1 son considerados como niveles altos, y en consecuencia, denota los niveles de fiabilidad de los instrumentos elaborados.

\section{RESULTADOS}

Los resultados de la muestra (16 estudiantes) sobre la metodología del trabajo colaborativo, se presentan siguiendo la dimensión construcción colaborativa del conocimiento y que cumple con el objetivo planteado: conocer la apreciación acerca de la inclusión de actividades de aprendizaje bajo el enfoque del trabajo colaborativo, mediante el uso de las TIC. Con respecto al género estuvo conformado por sexo masculino $56.3 \%$ y femenino $43.8 \%$, de tal manera que al cuestionarles si tenían experiencia previa en actividades de tipo colaborativas el $68.8 \%$ afirmó no tener experiencia contra el $31.3 \%$ que respondieron que sí. 
Los datos obtenidos en relación con la percepción (véase Tabla 2) y de acuerdo a las medias obtenidas, señalan una valoración positiva de los estudiantes que consideran adecuado el empleo de tareas basadas en el trabajo colaborativo. Además destacan, el grado de empatía considerada cómo la capacidad para comprender a los otros, esto se vio reflejado en el establecimiento de los objetivos en común con el grupo. Del mismo modo, el alumnado ha mostrado el acuerdo en los compromisos con los objetivos y consenso en el grupo.

\begin{tabular}{|l|c|c|c|}
\hline $\begin{array}{l}\text { ¿En qué grado son importantes los siguientes } \\
\text { aspectos en la construcción colaborativa del } \\
\text { conocimiento? }\end{array}$ & Media & $\begin{array}{c}\text { Desviación } \\
\text { Estándar }\end{array}$ & Varianza \\
\hline El compromiso con los objetivos de la tarea & 3.69 & .479 & .229 \\
\hline El compromiso con los acuerdos del grupo & 3.56 & .512 & .263 \\
\hline $\begin{array}{l}\text { Participación en los objetivos en común con el } \\
\text { grupo }\end{array}$ & 3.50 & .516 & .267 \\
\hline Reconozco las aportaciones de los demás & 3.81 & .403 & .163 \\
\hline El respeto a la diversidad & 3.88 & .342 & .117 \\
\hline Empatía: capacidad de comprender a los otros & 3.50 & .632 & .400 \\
\hline $\begin{array}{l}\text { Crítica constructiva de los diferentes puntos de } \\
\text { vista de los demás }\end{array}$ & 3.81 & .403 & .163 \\
\hline Manejo asertivo del error & 3.69 & .479 & .229 \\
\hline
\end{tabular}

Tabla 2. Resultados de la dimensión "percepción". Elaboración propia.

Por otro lado y con base en los resultados de las medias obtenidas (ver Tabla 3), destaca una actitud positiva respecto al uso de las TIC. Esto se comprueba, puesto que los estudiantes consideran que las herramientas digitales son importantes para su aprendizaje. En este sentido los alumnos entienden al trabajo colaborativo como una metodología que les permite compartir ideas y conocimientos sobre un determinado tema. Asimismo, se aprecia una motivación respecto al trabajo grupal, fundamentados en la responsabilidad individual y del grupo, para el logro de la tarea compartida.

\begin{tabular}{|l|c|c|c|}
\hline $\begin{array}{l}\text { Actitudes respecto al trabajo colaborativo con } \\
\text { TIC }\end{array}$ & Media & $\begin{array}{c}\text { Desviación } \\
\text { Estándar }\end{array}$ & Varianza \\
\hline $\begin{array}{l}\text { Considero que las herramientas digitales son } \\
\text { importantes para mi aprendizaje en el momento } \\
\text { actual. }\end{array}$ & 3.43 & .629 & .396 \\
\hline Me siento a gusto realizando tareas grupales. & 3.43 & .629 & .396 \\
\hline $\begin{array}{l}\text { La responsabilidad individual y en equipo es } \\
\text { positiva para el logro de la tarea. }\end{array}$ & 3.75 & .447 & .20 \\
\hline $\begin{array}{l}\text { La crítica en el grupo me ayudo a mejorar mis } \\
\text { aportaciones. }\end{array}$ & 3.56 & .813 & .663 \\
\hline $\begin{array}{l}\text { Las herramientas sociales no me favorecen en el } \\
\text { trabajo en grupo. }\end{array}$ & 1.43 & .629 & .396 \\
\hline Me agobia la participación en foros. & 1.75 & 1.06 & 1.133 \\
\hline
\end{tabular}




\begin{tabular}{|l|c|c|c|}
\hline $\begin{array}{l}\text { Me parece conveniente introducir la } \\
\text { colaboración en las actividades de aprendizaje. }\end{array}$ & 3.75 & .447 & .341 \\
\hline $\begin{array}{l}\text { Me motiva el trabajo grupal, siempre aprendo } \\
\text { de las experiencias de otros. }\end{array}$ & 3.87 & .341 & .117 \\
\hline
\end{tabular}

Tabla 3. Resultados de la dimensión "actitud". Elaboración propia.

Respecto a su proceso de aprendizaje, es bastante positivo el número de alumnos que considera que el desarrollo o implementación de este tipo de metodologías ha facilitado la comprensión de los temas didácticos adquiridos en clase y puestos en marcha al momento de articular los aspectos teóricos con las prácticas realizadas de manera colectiva. Por el contrario, son pocos los que consideran que las herramientas sociales no les aportan nada en el trabajo en grupo, así como los que consideran que la participación en los foros les agobia. Sin embargo, se destaca una opinión favorable acerca de la crítica constructiva, propiciada por el intercambio de punto de vista de sus compañeros, permitiendo con ello mejorar las habilidades de comunicación al momento de realizar las prácticas de manera grupal. En cuanto a su experiencia como estudiante con respecto al trabajo colaborativo, sus expresiones fueron las siguientes:

“...Ser estudiante 2.0 implica la multiculturalidad, es decir el respeto a la raza, religión, preferencia social, etc. y el trabajo colaborativo enriquece profesional y personalmente. De ahí que esta experiencia resultó muy enriquecedora...", (A1).

“...es grato saber y conocer las experiencias del otro y complementar las actividades para realizar un proyecto o trabajo...", (A16).

“...Como estudiante, el trabajo colaborativo siempre ha estado presente. En este sentido, las experiencias y los conocimientos compartidos han sido fundamentales para alcanzar los objetivos propuestos...", (A15).

"...Me gusta colaborar con las actividades ya que gusta compartir los conocimientos que tengo para poder ayudar a los demás y poder trabajar en armonía...", (A4).

Con respecto a la facilidad de uso del software PT en la modalidad multiusuario, el $85 \%$ de los encuestados, afirma que realizar las prácticas bajo esta modalidad presentó un uso sencillo, práctico y novedoso. Algunos de los comentarios por parte de los participantes fueron los siguientes:

“...Me gustó la forma de utilizar el PT en la modalidad multiusuario, ya que te permite conectar tu computadora personal (PC) con la de los demás compañeros...", (A9).

“...El trabajo realizado con la herramienta multiusuario, fue muy productivo, nos permitió trabajar de forma colaborativa, conectar las computadoras entre nosotros y realizar escenarios cercanos a la realidad...", (A3). 
“...Al principio no le entendía bien la modalidad multiusuario de Packet Tracer, solo fue cuestión de practicar y me pareció interesante el realizar las conexiones de mis topologías con las de mi compañeros...", (A1).

\section{CONCLUSIONES}

La discusión de este estudio, se enfoca en la aplicación del enfoque del trabajo colaborativo mediante el uso de las TIC en el alumnado universitario, y se sitúo en:

a) Articulación de la teórica con la práctica.- Un aspecto fundamental para la realización del diseño y configuración de las topologías de red, precisamente es la adquisición de los principios básicos en el manejo de los equipos de comunicaciones y de red. Esto se realizó mediante el uso del programa de cómputo denominado Packet Tracer, que permitió que los estudiantes diseñaran los escenarios de conexión y a su vez aplicarán la configuración en los equipos de red.

b) El trabajo colaborativo.- En lo que se refiere a las didácticas empleadas, se concentraron los estudiantes en la realización de los foros de discusión en donde el eje central del debate, se centró en la resolución de problemas en los escenarios de red planteados. De igual manera en las prácticas realizadas los estudiantes aplicaron la configuración vista en PT pero con equipo especializado de red, lo que permitió crear un escenario cercano a la realidad.

A manera de síntesis, se afirma que los estudiantes de la asignatura de Redes II, muestran una actitud y percepción favorable acerca del trabajo colaborativo. En general, entienden este enfoque como un trabajo en grupo, en donde las actividades son repartidas de manera equitativa y cada participante hace su mejor esfuerzo para la realización de la misma. Cabe destacar, que a pesar de que en el grupo contaban con conocimientos previos en cuanto a la configuración básica de los equipos de red, dichas actividades fueron realizadas de manera individual. Sin embargo, al cursar la presente asignatura, la dinámica fue realizar las prácticas en el modo "multiusuario", esto les permitió crear escenarios de red de manera colectiva y realizar el trabajo colaborativo.

Otro aspecto importante, fue el debate realizado en los foros de discusión, dicho espacio se ocupó como canal de comunicación para debatir los puntos vistos en clase. Esta situación, es compatible con estudios realizados con anterioridad por los autores Bassilotta y Herrada (2013). Al respecto Romero y Guitert (2012) señalan que la generación de ideas resulta favorable en ambientes colaborativos mediados por TIC en comparación con ambientes presenciales. Esto se confirmó, al momento de trabajar con la modalidad multiusuario en PT, toda vez que tenían que realizar las configuraciones de manera colaborativa.

Por otra parte, se puede señalar que la percepción y la actitud hacia el trabajo colaborativo por parte de los participantes resultó positiva. En las prácticas realizadas con la herramienta PT, los 
estudiantes pusieron énfasis al conectar en red las computadoras entre sus compañeros para después realizar la configuración que tenían asignada. Esto permitió llevar a un entorno real las actividades realizadas en la computadora, así como articular la teoría con las prácticas en el laboratorio de redes utilizando el equipo designado para ello.

Con base en los resultados presentados, se concluye que en el grupo de estudiantes evaluados existe una valoración positiva respecto a las actitudes acerca del trabajo colaborativo mediado con TIC, es claro que el uso de las aplicaciones digitales les permitió los participantes la creación de un entorno colectivo.

Finalmente, cabe precisar que es necesario en la actualidad se vive en una sociedad interconectada y según el reporte de Cisco (2014) el Internet viene a potenciar la economía global, para ello se requiere de profesionistas altamente capacitados en la configuración y administración de redes de datos, pero también en habilidades de colaboración que le faciliten la interacción con sus pares tanto de manera presencial como virtual. Por ello se considera que el estudiante tiene que estar preparado tanto con los sustentos teóricos como los prácticos, estos últimos deben de ser focalizados por las habilidades de colaboración que demandan los empleadores.

\section{REFERENCIAS}

AULA(2009). Elementos clave del proyecto AULA. Universidad Veracruzana. Fecha de acceso el 1 de Noviembre de 2012. Disponible en: www.uv.mx/aula

BISQUERRA, R. (2004). Metodología de la investigación educativa. Madrid: La Muralla.

BASILOTTA, V. y HERRADA, G. (2013). Aprendizaje a través de proyectos colaborativos con TIC. Análisis de dos experiencias en el contexto educativo. EDUTEC, Revista Electrónica de Tecnología Educativa. Núm.44, junio 2013.

CISCO (2014). El Internet de todo. Recuperado de:

http://share.cisco.com/loESocialWhitepaper/index-es.php\#/

DOMINGO, A. (2005). TIC, Internet, innovación y cambio educativo: estudios de caso. Universidad Oberta de Catalunya. Fecha de acceso el 28 de Octubre de 2012, Disponible en: http://www.ouc.edu/in3/dt/esp/domingo0605.html

ECHAZARRETA, C., PRADOS, F., POCH, J. y SOLER, J. (2009). La competencia El trabajo colaborativo: una oportunidad para incorporar las TIC en la didáctica universitaria. Descripción de la experiencia con la plataforma ACME (UdG). Universidad Oberta de Catalunya. No. 8. 2009, ISSN 1885-1541. 
HASSANDOUST, F. Y KAZEROUNI, M. (2009). Implications knowledge sharing through ECollaboration and Communicaction Tools. Journal of Knoledge Management, Economics and Information Technology, ISSN 2069-5934

JOHNSON, D. y JOHNSON, R. (1986). Positive interdependence, academic and collaborative-skills group contingencies, and isolated students. American Educacional Research Journal, 23, 476-488

ORTA, M. y OJEDA, A. (2010). Retos de la incorporación de las tecnologías de la información y comunicación en los procesos educativos. Innova Cesal. Fecha de acceso el 1 de Noviembre de 2012 Disponible en: http://www.innovacesal.org/innova_public docs01 innova/ic publicaciones 2012/indice.h $\underline{\mathrm{tm}}$

PEÑALOSA, E., GARCÍA, C. y ESPINOSA, M. (2008). La argumentación como recurso para el aprendizaje colaborativo en internet: una propuesta. Universidad Autónoma de México.

RAYÓN, L. (2005). La colaboración como contexto social de aprendizaje: la construcción y recreación compartida del conocimiento en entornos virtuales. Revista ciencias de la educación. Fecha de acceso 4 de Octubre de 2012. Disponible en: http://spdece.uah.es/papers/Rayon Final.pdf

ROMERO, M. y Guitert, M. (2012). Diseño y utilización de un entorno de aprendizaje colaborativo basado en la Web 2.0. Revista Latinoamericana de Tecnología Educativa. ISSN 1695-288X Vol. 11 No.1.

ROSELLI, N. (2011). Teoría del aprendizaje colaborativo y teoría de la representación social: convergencias y posibles articulaciones. Revista Colombiana de Ciencias Sociales. ISSN 2216-1201, No. 3, Diciembre 2011.

ROTSTEIN, B. (2006). El trabajo colaborativo en entornos virtuales de aprendizaje. Revista cognición No. 7, Octubre 2006. ISSN 1850-1974

SIEMENS, G. (2005). Knowing Knowledge. Fecha de acceso 2 de Septiembre de 2012, Disponible en www.knowingknowledge.com/book.php

VILLALUSTRE, L. y DEL MORAL, M. (2010). Evaluación del trabajo colaborativo virtual del Gameproyect de Ruralnet por los estudiantes universitarios. Revista DIM: Didáctica, Innovación y Multimedia, no18. ISSN 1699-3748.

VILLALUSTRE, L. y DEL MORAL, M. (2011). Competencias genéricas desarrolladas por los estudiantes con las e-actividades de Ruralnet. Aula Abierta, 39 (2). 35-46. ISSN 0210-2773

VILLALUSTRE, L. y DEL MORAL, M. (2012). Estrategias innovadoras de explotación de la Web 2.0 para un aprendizaje en red exitoso. En Bao, R. \& Flores, J., (Coord.). Las organizaciones 
virtuales y la evolución de la Web. Lima, Perú: Universidad de San Martín de Porres y Fondo Editorial.

Para citar este artículo:

Soto-Ortiz, J. L.; \& Torres-Gastelú, C. A. (2015). Apreciaciones sobre el diseño colaborativo de redes de datos mediante TIC. EDUTEC, Revista Electrónica de Tecnología Educativa, 51. Recuperado el dd/mm/aa de http://www.edutec.es/revista 